\title{
In-spiraling Clumps in Blue Compact Dwarf Galaxies
}

\author{
Bruce G. Elmegreen \\ IBM Research Division, T.J. Watson Research Center, 1101 Kitchawan Road, Yorktown \\ Heights, NY 10598 \\ bge@watson.ibm.com \\ Hong-Xin Zhang \\ Lowell Observatory, 1400 West Mars Hill Road, Flagstaff, Arizona 86001 USA; Purple \\ Mountain Observatory, Chinese Academy of Sciences, 2 West Beijing Road, Nanjing \\ 210008 China; Graduate School of the Chinese Academy of Sciences, Beijing 100080, China \\ Deidre A. Hunter \\ Lowell Observatory, 1400 West Mars Hill Road, Flagstaff, Arizona 86001 USA
}

\begin{abstract}
Giant star-formation clumps in dwarf irregular galaxies can have masses exceeding a few percent of the galaxy mass enclosed inside their orbital radii. They can produce sufficient torques on dark matter halo particles, halo stars, and the surrounding disk to lose their angular momentum and spiral into the central region in 1 Gyr. Pairs of giant clumps with similarly large relative masses can interact and exchange angular momentum to the same degree. The result of this angular momentum loss is a growing central concentration of old stars, gas, and star formation that can produce a long-lived starburst in the inner region, identified with the BCD phase. This central concentration is proposed to be analogous to the bulge in a young spiral galaxy. Observations of star complexes in five local BCDs confirm the relatively large clump masses that are expected for this process. The observed clumps also seem to contain old field stars, even after background light subtraction, in which case the clumps may be long-lived. The two examples with clumps closest to the center have the largest relative clump masses and the greatest contributions from old stars. An additional indication that the dense central regions of BCDs are like bulges is the high ratio of the inner disk scale height to the scale length, which is comparable to 1 for four of the galaxies.
\end{abstract}


Subject headings: Galaxies: bulges — Galaxies: evolution — galaxies: irregular - Galaxies: starburst - galaxies: individual (Mrk 178, DDO 155, Haro 29, NGC 2366, NGC 4861)

\section{Introduction}

Blue Compact Dwarfs (BCDs) are small galaxies with intense emission-lines from starburst HII regions in their central regions (Sargent \& Searle 1970). They are gas-rich like other dwarf irregulars (Chamaraux 1977; Gordon \& Gottesman 1981; Thuan \& Martin 1981), but much more centrally concentrated in stars (Noeske et al. 2003; Hunter \& Elmegreen 2006), star formation (Heller et al. 2000; Hunter \& Elmegreen 2004), and gas (Taylor et al. 1994; van Zee et al. 1998b). This concentration suggests that gas inflow following angular momentum loss led to enhanced star formation in a dense and gravitationally unstable central disk (Taylor et al. 1994; Papaderos et al. 1996; van Zee et al. 2001). Gas loss from the outer parts also produces a shrinking radius for star formation, as observed in dwarfs (Zhang et al. 2011; Koleva et al. 2011).

Individual star-forming regions in BCDs are relatively large, giving the galaxies a clumpy, irregular appearance in $\mathrm{H} \alpha$ (e.g. Kunth et al. 1988; Cairós et al. 2009b) and FUV (Thuan et al. 1997). Clump emission lines are supersonic and apparently virialized (Terlevich \& Melnick 1981; Firpo et al. 2011), which implies the clumps could last for several internal crossing times. Low velocity shear (Thuan et al. 1999; van Zee et al. 2001; Ramya et al. 2011) and resolved stellar population studies (Dohm-Palmer et al. 1998) also suggest the clumps could be long-lived, $100 \mathrm{Myr}$ or more. McQuinn et al (2010) and Zhang et al. (2011) suggest the starburst itself can last for $\sim 1$ Gyr.

Surrounding many BCDs are pools of HI, sometimes as large as 4 or more optical radii (Brinks \& Klein 1988; Tavlor et al. 1996; van Zee et al. 1998b; Putman et al. 1998; Pustilnik et al. 2001; Hoffman et al. 2003). The peripheral HI is often not simply rotating (van Zee et al. 1998b) or even in a disk (Pustilnik et al. 1997, 2001). This peripheral gas led to suggestions about cloud impacts (Gordon \& Gottesman 1981), weak interactions (Brinks \& Klein 1988; Pustilnik et al. 2002; Bravo-Alfaro et al. 2004), merging (Bekki 2008), and tidal effects (van Zee et al. 1998b; Pustilnik et al. 2001) in efforts to explain the high star formation rates. Alternatively, the HI could be vestigial streams or pools of cosmological accretion (Taylor et al. 1993; Thuan \& Izotov 1997; van Zee et al. 1998a; Wilcots \& Miller 1998), like the streams modeled for higher-mass galaxies (e.g., Ceverino et al. 2010). 
BCDs resemble young galaxies in many respects (Elmegreen et al. 2009b; Izotov et al. 2011; Griffith et al. 2011). They are gas-rich, low-metallicity (Izotov et al. 2001), relatively turbulent (van Zee et al. 1998b, 2001; Silich et al. 2002; García-Lorenzo et al. 2008), and highly clumped with locally intense star formation. They tend to lie at the edges of galactic clusters or in voids with only low-mass neighbors, and where harsh environmental effects like ram pressure stripping are minimal (Grogin \& Geller 2000; Drinkwater et al. 2001; Pustilnik et al. 2002). Those with the lowest metallicity tend to be relatively young, having formed most of their stars within the last few Gyrs (Searle \& Sargent 1972; Noeske et al. 2000; Johnson et al. 2000; Fricke et al. 2001; Papaderos et al. 2008). BCDs with less extreme metallicities tend to have relatively more old stars (e.g., Thuan 1983; Loose \& Thuan 1986; Crone et al. 2002; Caon et al. 2005; Cairós et al. 2007, 2009a.,b; Zhao et al. 2011; Zhang et al. 2011). In the most low-metal BCDs, there is little evidence for stars older than $\sim 200-500$ Myr; these include SBS 1415+437 (Thuan et al. 1999; Guseva et al. 2003a), SBS 1129+576 (Guseva et al. 2003b), and I Zw 18 (Papaderos et al. 2002).

Specific examples of BCDs illustrate these points. I Zw 18 is in many respects a morphologically young system. It has two giant star-forming regions inside a kpc-scale blue continuum of stars, ionized emission with an overall exponential profile (Papaderos et al. 2002), and an extensive HI envelope (van Zee et al. 1998a). Inside each region the star formation is widely distributed (Hunt et al. 2005). The rotation curve is flat but steeply rising in the inner part, where baryons may dominate dark matter (Lelli et al. 2011). Radial motions in the disk of $\sim 15 \mathrm{~km} \mathrm{~s}^{-1}$ suggest a major disturbance, which Lelli et al. (2011) suggest is tidal because there is a dwarf companion galaxy and elongated peripheral HI gas. The rotation curve gives a mass of $10^{8} M_{\odot}$ in which $\sim 70 \%$ is neutral gas (Contreras Ramos et al. 2011). It has very low metallicity (2\%-3\% solar, Izotov et al. 2001) even though old stars are present. Recchi et al. (2004) and others have considered the selective removal of metals by winds.

Another well-studied example is VII Zw 403, which is among the nearest BCDs to the sun, having a distance of 4.5 Mpc (Lynds et al. 1998). VII Zw 403 has a half-dozen big clumps and many $\mathrm{H} \alpha$ filaments from superbubbles (Lozinskava et al. 2006) in the midst of a smooth elliptical background of old red giant stars (Schulte-Ladbeck et al. 1998). The dynamical mass is $2 \times 10^{8} M_{\odot}$, with approximately $20 \%$ in HI (Thuan \& Martin 1981). The rotation speed is only $\sim 15 \mathrm{~km} \mathrm{~s}^{-1}$ (Simpson et al. 2011) and the metallicity is $5 \%$ solar (Martin 1997; Izotov et al. 1997). Lynds et al. (1998) used resolved stellar populations to date a major star burst to $600 \mathrm{Myr}$ ago, when the star formation rate was $\sim 30$ times higher than it is today. The current burst produced $\sim 10^{6} M_{\odot}$ within the last 10 Myr (Silich et al. 2002). 
The most intense star formation in BCDs can occupy very compact regions with extreme densities and local formation rates. SBS 0335-052 is a pair of extremely young interacting dwarf galaxies (Pustilnik et al. 2001; Ekta et al. 2009) without much of an underlying old population (Papaderos et al. 1998), and with a metallicity of 2.5\%-4\% solar (Izotov et al. 2009a; Peimbert \& Peimbert 2010). There are 6 super star clusters (Thuan et al. 1997), of which two, within $\sim 200$ pc of each other, have extremely intense star formation. Hunt et al. (2005) and Johnson et al. (2009) found radio free-free absorption and a very high emission measure where the electron density is $\sim 10^{3}-10^{4} \mathrm{~cm}^{-2}$, the star formation rate is $\sim 1 M_{\odot}$ $\mathrm{yr}^{-1}$, and the excitation comes from the equivalent of $\sim 10^{4} \mathrm{O} 7$ stars.

Here we propose that central accretion and long-lived starbursts in some BCDs arise from gravity-driven motions and torques produced by clump formation, clump dynamical friction, and clump interactions - the same processes that could make bulges in larger galaxies (e.g. Noguchi 1999; Immeli et al. 2004; Bournaud et al. 2007). BCDs have steep stellar profiles in the inner 500 pc that are exponential (Hunter \& Elmegreen 2006) or deVaucouleur's (Doublier et al. 1999), as in the bulges of earlier Hubble types. Such high central concentrations require baryonic mass inflow and significant angular momentum redistribution in the disk. Much of this inflow could have occurred when BCDs were young, but some of today's BCDs still look dynamically young even if there are old stars, and significant inflow could be occurring now.

Our emphasis differs from that in Governato et al. (2010), where simulations of dwarf galaxies highlight the removal of gas in order to avoid central concentrations. In these simulations, diffusion, torques, and pressure-driven inflows return some of this gas to the center (e.g., Recchi \& Hensler 2006; Dalcanton \& Stilp 2010), only to have it removed again by the next starburst, cycling in and out many times (e.g. Stinson etal. 2007; Revaz et al. 2009). The result is a bulge-free late-type galaxy and a time-changing central potential that converts a primordial dark matter cusp into a more uniform dark matter core (Read \& Gilmore 2005). The degree of this conversion varies for different simulations (Ogiva \& Mori 2011; Oh, et al. 2011). Alard (2011) note that the least evolved galaxies, having the highest relative gas abundances, tend to have the steepest inner density profiles, supporting the idea that gas recycling and star formation make the inner profiles shallow over time. These observations could imply that some BCDs still have steep central dark matter profiles, if these galaxies are relatively young. Del Popolo (2011) also model low-mass galaxies and suggest that tidal torques and the baryon fraction before collapse influence the central density profile of dark matter. Steeper central profiles are predicted to occur in more remote galaxies and in those with higher dark matter fractions; BCDs could be in this category too (Grogin \& Geller 2000). 
BCDs are unusual in having both a central concentration and a high gas abundance. This combination also appeared in massive galaxies at redshift $z \sim 2$ (Elmegreen et al. 2009a). Observations at intermediate-to-high redshift indicate that Hubble types arise mostly since $z \sim 1$ (Papovich et al. 2005; Bundy et al. 2006). The morphology seems to depend on dynamical maturity. High-mass disks like Hubble type Sa tend to be higher density than low-mass disks like Hubble type Sd (Roberts \& Haynes 1994). Thus, Sa's evolve more quickly to a centrally concentrated state with a low gas fraction and a low specific star formation rate (Sandage 1986; Zhang \& Buta 2007). If this trend of increasing central concentration and decreasing specific star formation rate continues into the future for lowmass galaxies, then some dwarf Irregulars might also evolve to a centrally concentrated state with little remaining gas. Mass loss from winds and supernovae in the low potential well of the dwarf could prevent such a central concentration however, depending on the relative rates of inflow from torques and outflow from winds. Those with dominant inflows could go through the BCD phase, as discussed here. Strong inflow depends on the presence of relatively massive clumps or tidal arms.

In what follows, we estimate the accretion time of a clumpy disk from dynamical friction (Sect. 2), and then consider whether observed clumps and other irregularities in BCDs are massive enough to drive significant disk evolution on a Gyr time scale (Sect. 3). A summary is in Section 4.

\section{Clump Accretion in BCDs}

\subsection{The Case with a few Giant Clumps}

The process of clump drag and interaction leading to coalescence in the center of a galaxy has been illustrated with detailed simulations of high redshift galaxies (Elmegreen et al. 2008; Ceverino et al. 2010). The clumps in these simulations formed spontaneously in a turbulent disk and had masses of about $5 \%$ of the total galaxy mass. Migration to the center took only

a few orbit times $(\sim 0.5 \mathrm{Gyr})$. Clump destruction by star formation feedback (Genel et al. 2010) does not stop the torques and accretion if each destroyed clump is replaced by a new one. This replacement is likely as long as the conditions for forming the first clumps, such as high gas fractions and turbulent speeds, are still present. A low ratio of turbulent speed to orbit speed would stop this process, because then the clumps that form by gravitational instabilities are relatively low-mass and produce proportionally weaker torques. The biggest star-forming regions in the Milky Way are only $\sim 10^{-3}$ times the disk mass and should have little tendency to move to the center. 
Dynamical friction and clump torques are important if the ratio of the disk Jeans mass to the galaxy mass is more than a few percent. This ratio scales with the square of the ratio of the gas velocity dispersion to the rotation speed. In high redshift galaxies, the rotation speed is normal for a massive disk but the dispersion is abnormally high (Erb et al. 2006; Förster Schreiber et al. 2011, and references therein) making the ratio high. In local dwarf Irregulars, the gas dispersion is normal for local galaxies, $\sim 10 \mathrm{~km} \mathrm{~s}^{-1}$, but the rotation speed is low, $\sim 50 \mathrm{~km} \mathrm{~s}^{-1}$ or less. In both cases the ratio of speeds is high and the clumps that form by gravitational instabilities are massive compared to the disk. The same processes of massive clump formation and angular momentum exchange should happen in high redshift galaxies and local gassy dwarfs because both have relatively large velocity dispersions compared to rotation speeds.

The timescale for dynamical friction between an orbiting clump of mass $M_{\mathrm{c}}$ in the disk and non-rotating dark matter particles or stars in a halo is $v(d v / d t)^{-1}$ where

$$
\frac{d v}{d t}=\frac{4 \pi \ln \Lambda G^{2} M_{\mathrm{c}} \rho}{v^{2}}\left(\operatorname{erf}[X]-\frac{2 X}{\pi^{1 / 2}} e^{-X^{2}}\right)=\frac{4 \pi \ln \Lambda G^{2} M_{\mathrm{c}} \rho \xi}{v^{2}}
$$

(Binney \& Tremaine 2008). Here, $v$ is the clump orbital speed, $X=v /\left(2^{1 / 2} \sigma\right)$ for halo 3D velocity dispersion $\sigma, \rho$ is the halo density, $\ln \Lambda$ is the coulomb factor, and $\xi$ is the quantity in parentheses. This formula assumes that the clump is a self-gravitating object surrounded by a uniform density of low-mass field stars or dark matter particles that have a Maxwellian velocity distribution function.

It is convenient from an observational point of view to write the local rotation speed as a power of the local radius, $v(r) \propto r^{\beta}$, since $\beta$ comes from the rotation curve. Starting with $\rho(r)=\rho_{0} r^{-\alpha}$ and $v(r)^{2}=G M_{\text {dyn }}(r) / r$, we get $v(r)^{2} /\left(4 \pi \rho[r] G r^{2}\right)=1 /(3-\alpha)=1 /(1+2 \beta)$. Then the dynamical friction time, $v /(d v / d t)$, in units of the dynamical orbit time, $r / v$, is

$$
T(r) \equiv \frac{v^{2}}{r(d v / d t)}=\frac{1}{\ln \Lambda \xi(1+2 \beta)} \times \frac{M_{\mathrm{dyn}}(r)}{M_{\mathrm{c}}}=T_{0}(r) \frac{M_{\mathrm{dyn}}(r)}{M_{\mathrm{c}}}
$$

where $M_{\mathrm{dyn}}$ is the galaxy dynamical mass enclosed within the orbital radius of the clump.

Dwarf galaxies have nearly solid body rotation in the inner parts (Swaters et al. 2002). BCDs can have steeply rising rotation curves in the dense inner regions, and flatter rotation curves beyond that (e.g., van Zee et al. 1998b; Lelli et al. 2011). The BCDs we consider in Section 3 have approximately-linear rising rotation curves in the vicinity of the giant clumps, and some have flat rotation curves beyond that (e.g., NGC 2366; Thuan et al. 2004). Thus for the main starburst regions we can take $\beta \sim 1$ or slightly less. If $v \sim \sigma$, then $\xi \sim 0.20$. In that case, the dimensionless time coefficient in equation (2) is $T_{0}=0.56$ for typical $\ln \Lambda=3$ (see below). For a clump at $r=0.5 \mathrm{kpc}$ orbiting with $v=10 \mathrm{~km} \mathrm{~s}^{-1}, r / v=49 \mathrm{Myr}$ and the 
dynamical friction time in physical units is $27 M_{\mathrm{dyn}} / M_{\mathrm{c}}$ Myr. This means that a clump with a mass greater than 2.7 percent of the enclosed galaxy mass has $T<1$ Gyr.

Note that $M_{\text {dyn }}$ decreases with radius, while the normalization quantity, $r / v$, is constant for $\beta \sim 1$. Thus the timescale gets smaller as the clump moves in. Writing the rate of change of clump angular momentum as $d L / d t=M_{\mathrm{c}} r(d v / d t)$ for frictional deceleration in the azimuthal direction $d v / d t$, and setting this equal to $M_{\mathrm{c}} v(d r / d t)$ for circular speed $v$ and radial drift speed $d r / d t<<v$, we get $d r / d t=v / T(r)$. For $\beta=1, v \propto r$ and $M_{\text {dyn }} \propto r^{3}$ so $T(r) \propto r^{3}$ if $\Lambda$ and $\xi$ are constant. Then, the time to reach the center is $1 / 3$ the instantaneous $T$ in equation 2. If $\beta=1 / 2$, then $v \propto r^{1 / 2}$ and $M_{\text {dyn }} \propto r^{2}$ so $d r / d t \propto r^{-3 / 2}$ and it takes $0.4 T$ to reach the center.

One uncertainty in this result is the ratio of the disk orbit speed to the halo velocity dispersion, which enters into $\xi$. This ratio depends on whether the halo has a core or a cusp, and on the nature of the core. We consider two extreme cases: a Burkert (1995) halo density profile in the case of a core, and an NFW (Navarro et al. 1996) profile for a cusp. For the Burkert profile, $\rho(x)=\rho_{\mathrm{s}}\left([1+x]\left[1+x^{2}\right]\right)^{-1}$ with $x=r / r_{\mathrm{s}}$ and scale factors $\rho_{\mathrm{s}}$ and $r_{\mathrm{s}}$. We use this with the equation of hydrostatic equilibrium in the radial direction to determine $\beta$ and $v / \sigma$ as functions of $x$. Hydrostatic equilibrium implies $d P / d r=-G M(r) \rho(r) / r^{2}$ where $P=\rho \sigma_{1 D}^{2}$ for $1 \mathrm{D}$ dispersion $\sigma_{1 D}=\sigma / 3^{1 / 2}$ and $M(r)=\int_{0}^{r} 4 \pi r^{2} \rho(r) d r$. We assume the boundary condition $P \sim 0$ and $\sigma \sim$ constant at the edge of the halo, which is taken to be where $\rho=10^{-4} \rho_{0}$. Figure 1 shows $v / \sigma, \xi, \beta$, and $T_{0}$ as functions of position $x$ (determined by numerical integration). $\beta \sim 1$ for a solid body rotation, so $x$ must be small in the visible part of the disk. For example, $x=0.54$ at the half-density point, where $\rho=0.5 \rho_{0}$, and there $v / \sigma=0.49, \xi \sim 0.03, \beta=0.72$, and $T_{0} \sim 4.6$. For $r=0.5 \mathrm{kpc}$ and $v \sim 10 \mathrm{~km} \mathrm{~s}^{-1}$, the friction time is then $r T_{0} / v=220 M_{\text {dyn }} / M_{\mathrm{c}}$ Myr. This implies that a clump with a relative mass of $M_{\mathrm{c}} / M_{\mathrm{dyn}}=5 \%$ takes $\sim 1.5$ Gyr to spiral in, considering the factor of $1 / 3$ that accounts for a decreasing $M_{\text {dyn }}$ with radius, as discussed above.

The NFW dark matter profile is a little faster. For this, $\rho=\rho_{\mathrm{s}}\left(x[1+x]^{2}\right)^{-1}$ with $x=$ $r / r_{\mathrm{s}}$ again. There is a density singularity at the center that produces a logarithmic divergence of a quantity like pressure if the equation of hydrostatic equilibrium is considered (because $M(r) \rho / r^{2} \sim 1 / r$ near the center). We consider instead that the halo 3D velocity dispersion is comparable to the rotation speed (Navarro et al. 1996), $v \sim \sigma$, which gives $\xi=0.20$. Writing for the galaxy mass $M_{\text {dyn }}(x)=4 \pi r_{\mathrm{s}}^{3} \rho_{\mathrm{s}} \mathcal{M}(x)$, where $\mathcal{M}(x)=\ln (1+x)-x /(1+x)$, the slope of the rotation curve is now given by $1+2 \beta=x^{2} /\left(\mathcal{M}(x)(1+x)^{2}\right)$. Figure 1 shows $\beta$ as a dashed line. We see $\beta \sim 0.5$ for small $x$ and then $T_{0} \sim 0.83$ with $\xi=0.2$. This is a smaller prefactor than for a cored halo because of the higher $v / \sigma$. The dynamical friction time for an NFW halo is $\sim 41 M_{\mathrm{dyn}} / M_{\mathrm{c}}$ Myr with $r=0.5 \mathrm{kpc}$ and $v \sim 10 \mathrm{~km} \mathrm{~s}^{-1}$. Clumps 
with $M_{\mathrm{c}} / M_{\mathrm{dyn}}>1.6 \%$ take $<1$ Gyr to spiral in, considering the factor of $2 / 5$ that accounts for a decreasing $M_{\mathrm{dyn}}$ with radius when $\beta=0.5$.

Dwarf galaxies do not appear to have NFW halos at the present time (e.g., Zackrisson et al. 2006; van Eymeren et al. 2009; Kuzio de Narav 2009; Amorisco \& Evans 2012), although it is difficult to be certain (Spekkens et al. 2005; Valenzuela 2007) and it is not yet known whether BCDs differ from other dwarfs in this regard. An important point for dynamical friction is the relative velocity dispersion of the field particles, $\sigma / V$, which enters into $\xi$ as shown above. The validity of the Chandresekhar formula is also a question. Goerdt et al. (2010) modeled sinking massive objects like what we consider here and showed that a central cusp turns into a core inside the radius where the sinking mass equals the enclosed dark halo mass. Further sinking in their model stalled at this radius because of a decrease in dynamical friction in the core. This result was also found by Read et al. (2006) and others. In an detailed study of dynamical friction in cored galaxies, Inoue (2011) explained the loss of frictional forces as a result of orbit resonances that appear when the orbit time is independent of radius, as is the case for a constant central density. Another limitation is that after a bulge forms, tidal forces from the bulge can rip apart remaining clumps and prevent them from reaching the center (Elmegreen et al. 2008). These considerations make it plausible that in some BCDs today, giant clumps come in from larger radii and then stall at the edge of a dark matter core, building up the central region from inside out. Accretion to the center could have occurred more readily when the galaxies were young and the dark matter profiles were more cuspy.

Sánchez-Salcedo et al. (2006) considered a similar situation with in-spiraling globular clusters in dwarf galaxies. They concluded that dwarfs should have dark matter cores rather than cusps so that the globular clusters stall midway in the disk where they can still be seen. Galaxies with giant nuclear star clusters, however, may have allowed their disk clusters to reach the center (Böker 2010; Antonini et al. 2011; Hartmann et al. 2011). The resemblance of these galaxies to BCDs is compelling if we allow for a difference in the mass and size of the disk clumps that form by star formation: BCDs have relatively massive and large disk clumps that could spiral in to form massive and large central cores, while normal dwarfs and galaxies have relatively small star clusters, which could spiral in to form small nuclear clusters.

\subsection{Clumps with a Power-Law Mass Function}

As mentioned above, individual clumps need not survive the full trip to the center to drive accretion if new clumps replace dispersed clumps in a steady state. Similarly, there 
need not even be a single giant clump. Any irregularities moving through a slower rotating halo will have dynamical friction drag, and the total torque on the medium will depend on the mean squared mass of those irregularities. Consider the equations of disk accretion starting with the continuity equation in two dimensions (Pringle 1981):

$$
\frac{d}{d t} \Sigma+r^{-1} \frac{d}{d r} r \Sigma v_{\mathrm{r}}=0
$$

the surface density is $\Sigma$ and the radial drift velocity is $v_{\mathrm{r}}$. The torque equation is

$$
\frac{d}{d t} r \Sigma v+r^{-1} \frac{d}{d r} r \Sigma v_{\mathrm{r}} r v=\text { torque/area }
$$

where $v$ is again the azimuthal speed. For a viscous disk, the torque per unit area is $R^{-1} d G / d R$ where $G=\nu \Sigma A r^{2}$ for viscous coefficient $\nu$ (comparable to the product of the clump mean free path and the rms speed), and Oort rotation constant $A$, which is the rate of shear. We are not concerned with viscosity in this paper because $A$ is small for dwarf galaxies with little shear; $\nu$ is also usually small compared to dynamical torques in galaxy disks (however, see Wang et al. 2009). Here we consider dynamical friction between orbiting clumps in the disk and the halo and other parts of the disk. If an annular area has $N$ clumps of mass $M_{\mathrm{c}}$, then the total torque exerted on these clumps is $N M_{\mathrm{c}} r d v / d t$ for deceleration in the azimuthal direction $d v / d t$ from equation (11). This quantity depends on the mean squared clump mass because $d v / d t$ depends on clump mass. For clump mass function $d n\left(M_{\mathrm{c}}\right) / d M_{\mathrm{c}} \propto M_{\mathrm{c}}^{-\delta}$,

$$
<N M_{\mathrm{c}}^{2}>=\frac{2-\delta}{3-\delta} M_{\mathrm{c}, \max }<N M_{\mathrm{c}}>=f M_{\mathrm{c}, \max }<N M_{\mathrm{c}}>.
$$

The prefactor $f$ ranges between $f=0.33$ at $\delta=1.5$ and $f=0.06$ at $\delta=2$ (for $\delta$, see e.g. Heithausen et al. 1998). In the latter case, the integral over $M^{2} n(M)$ gives $\ln \left(M_{\mathrm{c}, \max } / M_{\mathrm{c}, \min }\right)$, whose value is $\sim 16$ for typical $M_{\mathrm{c}, \max } \sim 10^{7} M_{\odot}$ and $M_{\mathrm{c}, \min } \sim 1 M_{\odot}$. Because $\left\langle N M_{\mathrm{c}}\right\rangle$ /Area $=\Sigma$, we have $\left\langle N M_{\mathrm{c}}^{2}\right\rangle /$ Area $=f \Sigma M_{\mathrm{c}, \max }$. Then the torque/area for the above halo model becomes $f \Sigma r d v / d t=\Sigma \gamma / r$ where $\gamma=(1+2 \beta) f \ln \Lambda G M_{\mathrm{c}, \max } \xi$. For a fixed galactic potential ( $v$ independent of time), the torque equation is now

$$
r^{2} v \frac{d \Sigma}{d t}+\frac{d}{d r} r \Sigma v_{\mathrm{r}} r v=-\Sigma \gamma .
$$

We can simplify this by writing $\mu=r \sum v_{\mathrm{r}}$ and noting that $(d / d r) \mu r v=\mu(d / d r) r v+$ $r v d \mu / d r=\mu(d / d r) r v-r^{2} v(d \Sigma / d t)$ using the continuity equation. Then $d \Sigma / d t$ cancels in the torque equation and we get

$$
r \Sigma v_{\mathrm{r}} \frac{d r v}{d r}=-\Sigma \gamma
$$


This may be solved for $v_{\mathrm{r}}$ since everything else is a known function of $r$, and then the result can be put into the continuity equation to get $d \Sigma / d t$. Note that $d r v / d r=(1+\beta) r v$; for the other radial derivative, we set $d / d r \sim 1 / r$. The resultant normalized accretion timescale is

$$
T=\frac{v \Sigma}{r d \Sigma / d t} \sim \frac{(1+\beta)}{f \ln \Lambda \xi(1+2 \beta)} \times \frac{M_{\mathrm{dyn}}(x)}{M_{\mathrm{c}, \max }}=T_{1}(x) \frac{M_{\mathrm{dyn}}(x)}{M_{\mathrm{c}, \max }} .
$$

This time is larger than before by the ratio $(1+\beta) / f$. Setting $\beta=1, \ln \Lambda=3$ and $\xi \sim 0.03$ for a Burkert core, and taking $f \sim 0.1$, we get $T_{1}=74$ and a physical accretion time of $1.2 M_{\mathrm{dyn}} / M_{\mathrm{c}, \max }$ Gyr for $r=0.5 \mathrm{kpc}, v=10 \mathrm{~km} \mathrm{~s}^{-1}$ with the factor $1 / 3$ to account for a decreasing $M_{\text {dyn }}$ with radius. For a NFW core with $\beta=0.5$ and $\xi=0.2, T_{1}=12.5$ and the accretion time is $240 M_{\text {dyn }} / M_{\mathrm{c}, \max }$ Myr for $r=0.5 \mathrm{kpc}, v=10 \mathrm{~km} \mathrm{~s}^{-1}$ with the factor $2 / 5$. Now we see that it takes about 1 Gyr for $10 \%$ of the ISM to accrete to the center from the inner half-kpc if the largest cloud in a power law distribution of cloud masses is $12 \%$ and $2.4 \%$ of the enclosed galaxy mass for the Burkert and NFW profiles, respectively. If the largest cloud has a mass much larger than the extrapolation of a power law distribution from the other clouds, then the previous analysis for a single cloud applies.

\subsection{Clump-Clump Interactions}

Clump-clump interactions can also drive accretion by direct gravitational forces. The acceleration on one clump by another clump is $G M_{\mathrm{c}} / \Delta r^{2}$ for separation $\Delta r$. This acceleration cumulatively distorts the clump's motion until its velocity has changed significantly. The timescale for this change is $v$ divided by the acceleration, and in units of the orbit time, it is $v^{2} / r$ divided by the acceleration. Writing $v^{2} / r=G M_{\mathrm{dyn}} / r^{2}$, the normalized interaction time becomes $(\Delta r / r)^{2}\left(M_{\mathrm{dyn}} / M_{\mathrm{c}}\right)$. For big clumps, $\Delta r \sim r$, and the normalized interaction time is just the ratio of masses. That means $T_{0} \sim 1$ in an equation like (2), and the accretion time is $\sim 49 M_{\mathrm{dyn}} / M_{\mathrm{c}}$ Myr for $r=0.5 \mathrm{kpc}$ and $v \sim 10 \mathrm{~km} \mathrm{~s}^{-1}$.

Other torques will contribute to the inflow of gas, so $T$ is an upper limit based on dynamical friction with the halo. The disk also will produce a torque if there is shear, because then the clump will drive a spiral wake (Julian \& Toomre 1966) and the wake will have its own torque that drives mass inward (Lynden-Bell \& Kalnajs 1972). Small galaxies tend to have little shear, however. 


\section{Observations of Clump Properties in Dwarf Irregulars and BCD Galaxies}

\subsection{Clump Mass Fractions and Accretion Times}

Many of the observations referenced in the introduction concern BCD or other dwarf irregular galaxies that have relatively large gas velocity dispersions compared to the rotation speeds, relatively thick disks compared to the galactic radii, and relatively massive starforming regions compared to the galaxy masses. They are good candidates for the extreme torques and inward migrations discussed above. Five examples are given in Table 1, along with properties of their primary star-formation clumps. As above, the clump mass is denoted by $M_{\mathrm{c}}$, the galactocentric radius at the center of the clump is $r_{\mathrm{c}}$, and the galaxy mass inside the clump radius is $M_{\mathrm{dyn}}$, measured as $r_{\mathrm{c}} v\left(r_{\mathrm{c}}\right)^{2} / G$ for local rotation speed $v\left(r_{\mathrm{c}}\right)$. Also for reference, we give the total galaxy stellar mass, $M_{\mathrm{s}}$, the total baryonic galaxy mass (gas+stars), $M_{\mathrm{b}}$, the Coulomb factor $\Lambda \sim\left(R_{\text {clump }} / R_{\text {gal,tot }}\right)\left(R_{\text {gal,tot }} / r_{\mathrm{c}}\right)^{1+2 \beta}\left(M_{\text {dyn }} / M_{\mathrm{c}}\right)$, and the accretion time $T$ from equation (2) for NFW and Burkert dark matter profiles. The Coulomb factor comes from the approximate expression $\Lambda \sim\left(R_{\text {clump }} / R_{\text {gal,tot }}\right)\left(M_{\text {gal,tot }} / M_{\mathrm{c}}\right)$ in Binney \& Tremaine (2008), where $R_{\text {clump }}$ and $M_{\mathrm{c}}$ are the clump radius and mass, and $R_{\text {gal,tot }}$ and $M_{\text {gal,tot }}$ are the total galaxy radius and mass. We take $M_{\text {gal,tot }} / M_{\text {dyn }} \sim\left(R_{\text {gal,tot }} / r_{\mathrm{c}}\right)^{1+2 \beta}$ for rotation curve $v \propto r^{\beta}, R_{\text {clump }}$ equal to half the clump aperture in the table, and $R_{\text {gal,tot }} / r_{\mathrm{c}}$ from Figure 2. As discussed above, the NFW profile has $\beta=0.5, \xi=0.2$, and a time multiplier of 0.4 to account for faster accretion as the clump approaches the center; the Burkert profile has $\beta=1, \xi=0.03$, and a multiplier of 0.33 .

Color composite images are shown in Figure 2. For four galaxies, they are made with $\mathrm{U}$ and $J$ band images from Hunter \& Elmegreen (2006); for NGC 4861, they are made with $u$ and $\mathrm{z}$ band images from the Sloan Digital Sky Survey (SDSS; Stoughton et al. 2002). The elliptical contours outline the giant star-forming clumps that we consider to be candidates for inward migration. The cross marks the center of the galaxy as defined by the outer elliptical isophotes in $\mathrm{V}$ band.

The HI rotation curves for our galaxies are close to solid body in the clump region. They are not accurate enough to tell if the dark matter halo is cored or cuspy (which give rotation curve slopes $\beta=1$ or 0.5 in these two cases, respectively). To determine $v$ at the clump radius $r_{\mathrm{c}}$, we fit the observed rotation curve to a deprojected speed, $v_{\mathrm{t}}$, and radius, $r_{\mathrm{t}}$, at the limit of the observation or the turnover point, whichever comes first. Then the total galaxy mass inside the clump radius is taken to be $v_{\mathrm{t}}^{2} r_{\mathrm{c}}^{3} /\left(r_{\mathrm{t}}^{2} G\right)$, assuming $\beta=1$ for this. For the different galaxies, the values of $\left(v_{\mathrm{t}}, r_{\mathrm{t}}\right)$ in $\left(\mathrm{km} \mathrm{s}^{-1}, \mathrm{kpc}\right)$, are, DDO 155: (8, 0.15) (Carignan et al. 1990); Haro 29: (34, 1.7)(Stil \& Israel 2002); NGC 2366: (50, 3.3) (Hunter et al. 2001; Thuan et al. 2004); NGC 4861: (40, 3.3) (Thuan et al. 2004). 
For DDO 155 (also known as GR8), the HI observations by Begum \& Chengalur (2003) suggest that the velocity field is complex, so the dynamical galaxy mass is inaccurate. Lo, et al. (1993) and Begum \& Chengalur (2003) suggest the velocities have an expanding or contracting component, which Begum \& Chengalur (2003) fits to a peak value of 10 $\mathrm{km} \mathrm{s}^{-1}$, with some radial variations. They also fit the rotating part to a peak value of $6 \mathrm{~km}$ $\mathrm{s}^{-1}$ at the edge (the escape speed was estimated to be $\sim 30 \mathrm{~km} \mathrm{~s}^{-1}$ ). The velocity of the giant clump outlined in Figure 2 is smaller than the systematic velocity, so if it is on the near side of the galaxy, then it is expanding away from the center. Begum \& Chengalur (2003) consider an explosive origin for this motion but note the lack of old star clusters that might have driven this explosion; they suggest that HII regions might have had the necessary force. If the motion is inward, then Begum \& Chengalur (2003) suggest that the clumps might have coalesced to form the galaxy and are now dispersing to make a disk. They note that there are no tidal features, however. This inward moving interpretation is consistent with the model presented in the present paper; tidal features are not expected because the torques are generated internally. The timescale for inward motion given in Table 1 is $\sim 200 \mathrm{Myr}$ for DDO 155. This timescale is consistent with the results of Dohm-Palmer et al. (1998), who find from resolved stellar population studies that star formation lasts in each clump for $\sim 100$ Myr. They suggest that this long time requires gravitational self-binding of the clumps. Dohm-Palmer et al. also suggest that the clumps come and go on this time scale, with the current generation of clumps at the positions of the three main HI clouds. Thus the present model of massive clump formation by gravitational instabilities in a gas-rich galaxy, relatively long clump ages from modest gravitational self-binding, and angular momentum loss through halo, disk, and clump-interaction torques, is consistent with the HI and stellar observations and previous interpretations of DDO 155.

Mrk 178 does not have a published rotation curve, but the HI line width was given by Bottinelli et al. (1973), who also derived a total dynamical mass ("indicative mass") from the equation $M_{\text {tot.dyn }}=3 \times 10^{4} r_{\mathrm{H}} W^{2} M_{\odot}$ for Holmberg radius $r_{\mathrm{H}}$ in kpc and linewidth $W$ in $\mathrm{km} \mathrm{s}^{-1}$ (Bottinelli et al. 1968). Scaling to our distance, the Holmberg radius is $r_{\mathrm{H}}=2.9$ kpc and the total dynamical mass is $M_{\mathrm{H}}=1.6 \times 10^{9} M_{\odot}$. If we assume this rotation curve is solid body, then the dynamical mass inside radius $r_{\mathrm{c}}$ is $M_{\mathrm{dyn}}\left(r_{\mathrm{c}}\right)=M_{\mathrm{H}}\left(r_{\mathrm{c}} / r_{\mathrm{H}}\right)^{3}$. Setting $r_{\mathrm{c}}=0.39 \mathrm{kpc}$ from Table 1 , we get $M_{\mathrm{dyn}}\left(r_{\mathrm{c}}\right)=3.9 \times 10^{6} M_{\odot}$.

Clump and galaxy stellar masses were derived by fitting the SEDs over a range of passbands inside deprojected circular apertures (see Zhang et al. 2011). The aperture sizes were determined from the U-band brightness contours shown in Figure 2. Local background intensities came from larger annuli around the clumps and were subtracted from the clump intensities. For Mrk 178, DDO 155 and NGC 2366, the SEDs used observations in U, B, V, and J passbands (Hunter \& Elmegreen 2006). For Haro 29, we used only U, B and J bands 
from Hunter \& Elmegreen (2006) because the V-band photometry is inconsistent with the others. For NGC 4861, we used ugiz data from SDSS. The giant clump in NGC 2366 was also studied by Kennicutt et al. (1980). The giant clump in NGC 4861 is known as Mrk 59 and was studied by Izotov et al. (2009b) and others.

Although there is HI gas present in some of the clumps, and perhaps even molecular gas connected with current star formation, we do not include gas in the clump masses. In DDO 155, for example, the HI mass in the clump is a few times $10^{5} M_{\odot}$ (Carignan et al. 1990; Begum \& Chengalur 2003), which is comparable to the stellar mass. In NGC 2366, an HI cloud at the position of the clump contains several $\times 10^{5} M_{\odot}$ (Hunter et al. 2001), which is $\sim 20 \%$ of the stellar mass. Considering the possible addition of gas, the clump masses and mass fractions given in Table 1 are lower limits.

As part of the fits for clump mass, we also obtained crude star formation histories in the clumps (Zhang et al. 2011). These are determined as relative stellar masses younger than 0.1 Gyr, in the time interval between 0.1 Gyr and 1 Gyr, and older than 1 Gyr. For the five galaxies, the relative masses in the intervals $(<0.1 \mathrm{Gyr}, 0.1-1 \mathrm{Gyr},>1 \mathrm{Gyr})$ are, Mrk 178: (0.42, 0.49, 0.09), DDO 155: (0.11, 0.23, 0.66), Haro 29: (0.17, 0.15, 0.68), NGC 2366: $(0.28,0.5,0.22)$, and NGC 4861: $(0.27,0.50,0.23)$. Evidently the SEDs indicate significant clump components older than 1 Gyr even after background disk subtraction. The dominant appearance of these clumps in the J-band (Fig. 2) suggests the same thing. If these old massive components are really present, then they would have to be gravitationally bound to the clump and the clump would have to be long-lived. We note that gravitational instabilities in a disk of gas and stars can collect both gas and a significant mass of background field stars into a clump when the velocity dispersions and densities of the two components are similar (Elmegreen 2011). Background field stars also fall into the clump and get trapped because of the changing gravitational potential as its mass grows (Fellhauer et al. 2006). In our sample, the clumps that are relatively closest to the center (in Mrk 178, DDO 155 and Haro 29) contain the highest fraction of old stars. This suggests a larger total age for the more centralized clumps than for the more peripheral clumps, which is consistent with a history of inward migration.

The 9th column in Table 1 gives the ratio between the clump mass and the galaxy dynamical mass inside the clump radius. As shown in the previous sections, if this ratio is larger than a few percent, the clump could significantly perturb the surrounding disk and cold halo particles, leading to the loss of clump orbital angular momentum in less than $\sim 1$ Gyr. The tabulated mass fractions are in this range. The timescale for their migration is in the last column, assuming NFW and Burkert profiles in two cases, and using the observed rotation speed at the clump position. The mass fractions are higher and the timescales are 
smaller when the clumps are relatively close to the center, because only the inner parts of the galaxies are included in $M_{\mathrm{dyn}}$.

\subsection{Galaxy Thickness and Scale Length Ratios}

Clump accretion can thicken the central regions because the stellar orbital energy gets mixed into three dimensions during the final merger phase (Bournaud et al. 2007). What is important is the ratio of the disk scale height, $H=\sigma^{2} /(\pi G \Sigma)$, to the disk scale length $R_{\mathrm{d}}$. Here, $\sigma$ is the perpendicular velocity dispersion in the central region of the BCD, and $\Sigma$ is the central mass column density of the disk. For reference values $\sigma=10 \mathrm{~km} \mathrm{~s}^{-1}$ and $\Sigma=10 M_{\odot} \mathrm{pc}^{-2}$, we obtain $H=740$ pc. Most BCDs in Hunter \& Elmegreen (2006) have $R_{\mathrm{d}} \sim 500$ pc or less, so $H$ and $R_{\mathrm{d}}$ are comparable. This means the inner parts of BCDs are $3 \mathrm{D}$ objects like a bulge.

Detailed consideration of the BCDs in Table 1 confirm that the inner disk thicknesses are comparable to or larger than the inner disk scale lengths. Putting dimensions into the thickness equation, we get

$$
H=740\left(\sigma / 10 \mathrm{~km} \mathrm{~s}^{-1}\right)^{2}\left(\Sigma / 10 M_{\odot} \mathrm{pc}^{-2}\right)^{-1} \mathrm{pc} .
$$

Zhang et al. (2011) determined disk stellar mass densities and scale lengths from SED fits. Values are given in Table 2, The average $H / R_{\mathrm{d}} \sim 2.6$, so the BCDs in this study should have relatively thick inner regions. The ratio would be larger for larger perpendicular velocity dispersions - the assumed value of $10 \mathrm{~km} \mathrm{~s}^{-1}$ for stars seems to be a lower limit. We note that the BCDs with giant clumps closest to the center have the highest ratios of height to length.

Height-to-length ratios greater than unity in Table 2 are difficult to understand. They would be smaller if additional mass were in the disk. This suggests that some of the BCDs in our survey have a considerable mass column density of gas in the inner disk, perhaps comparable to or larger than the stellar column density. Such high masses of gas might be expected for the clumps in which the starbursts are occurring (e.g., larger than several hundred $M_{\odot} \mathrm{pc}^{-2}$ in molecules, which is typical for local giant molecular clouds), but there might also be a dense molecular and atomic intercloud medium where the average exceeds the average stellar value of $10 M_{\odot} \mathrm{pc}^{-2}$. Alternatively, a high filling factor of star-forming gas clumps that individually have mass column densities in excess of $\sim 100 M_{\odot} \mathrm{pc}^{-2}$ could produce an average gas column density in the inner part that exceeds the stellar column density. This could explain why the BCDs in Table 2 that have their massive clumps closest to the center also have the largest height-to-length ratios, i.e., these galaxies have higher $\Sigma$ than 
we assume because of contributions from molecular and dense atomic material in clumps. Massive clump accretion like that discussed here would drive significant gas accretion, not only in the clumps but also of the interstellar material between the clumps, which gets dragged along with the clumps by gravitational and magnetic forces.

The mass column densities of inner disk HI gas have been observed for most of these galaxies. For both DDO 155 and NGC 2366, it is $\sim 10 M_{\odot} \mathrm{pc}^{-2}$ (Carignan et al. 1990; Hunter et al. 2001). Haro 29 has a hole in the central HI but the clump is very close and it has an average column density of $\sim 20 M_{\odot} \mathrm{pc}^{-2}$ (Stil \& Israel 2002). NGC 4861 has a large HI concentration in the center with a column density of $\sim 30 M_{\odot} \mathrm{pc}^{-2}$ (Thuan et al. 2004). Some of these values are larger than the corresponding central stellar mass column density by a factor 2 or more, which lowers $H / R_{\mathrm{d}}$ in proportion. Further studies of the gas column densities in the centers of BCD galaxies should clarify their relative thicknesses.

\section{Summary}

Young stellar clumps that form by gravitational instabilities in a galaxy disk can have such a high mass relative to the enclosed galaxy mass that they produce dynamically significant torques on the halo stars and cold dark matter particles, on the disk, and on each other. If the clump mass fraction exceeds a few percent, then these torques can drive an inflow of the clump's amount of mass in less than 1 Gyr. This process has been suggested for the formation of bulges in disk galaxies at high redshift, but it may apply also to local clumpy galaxies. Because of the general tendency for downsizing, in which active star formation occurs in galaxies with ever smaller masses as the universe ages, the clumpy phase now is mostly limited to dwarfs. We suggest that BCDs are an example of a local clumpy star-bursting galaxy in which the clumps are large enough to drive significant accretion in a Gyr or less. This would explain the dense stellar inner disks of these galaxies, and the prolonged star formation near the center.

The BCDs in our sample also have relatively thick inner regions, reminiscent of bulges in spiral galaxies. They are even a little too thick if only the stellar surface densities are considered. This suggests there could be a dense atomic or molecular component in the inner region that has an average surface density comparable to or exceeding that from stars.

This work was funded in part by the National Science Foundation through grants AST0707563 and AST-0707426 to DAH and BGE. HZ was partly supported by NSF of China through grants \#10425313, \#10833006 and \#10621303 to Professor Yu Gao. We are grateful to the referee for helpful comments. 


\section{REFERENCES}

Alard, C. 2011, ApJL, 728, 47

Amorisco, N. C. \& Evans, N. W. 2012, MNRAS,m 419, 184

Antonini, F., Capuzzo-Dolcetta, R., Mastrobuono-Battisti, A., \& Merritt, D. 2011, arXiv:1110.5937

Begum, A., \& Chengalur, J.N. 2003, A\&A, 409, 879

Bekki, K., Freeman, K.C. 2002, ApJ, 574, L21

Bekki, K., 2008, MNRAS, 388, L10

Binney, J., \& Tremaine, S. 2008, Galactic Dynamics, Princeton University Press

Böker, T. 2010, in Star clusters: basic galactic building blocks throughout time and space, eds. R. de Grijs \& J. Lepine, IAU Symposium 266, 58

Bottinelli, L., Gouguenheim, J., Heidmann, J., \& Heidmann, N. 1968, AnAp, 31, 205

Bottinelli, L., Gouguenheim, L., \& Heidmann, J. 1973, A\&A, 22, 281

Bournaud, F., Elmegreen, B.G., \& Elmegreen, D.M. 2007, ApJ, 670, 237

Bravo-Alfaro, H., Brinks, E., Baker, A. J., Walter, F., \& Kunth, D. 2004, AJ, 127, 264

Brinks, E., \& Klein, U. 1988, MNRAS, 231, P63

Bundy, K., Ellis, R.S., Conselice, C.J., Taylor, J.E., Cooper, M.C., Willmer, C.N.A., Weiner, B.J., Coil, A.L., Noeske, K.G., Eisenhardt, P.R.M. 2006, ApJ, 651, 120

Burkert, A. 1995, ApJ, 447, L25

Cairós, L.M., Caon, N., Papaderos, P., Noeske, K., Vlchez, J.M.. Garca Lorenzo, B., MuñozTuñón, C. 2003, ApJ, 593, 312

Cairós, L.M., Caon, N., Garca-Lorenzo, B., Monreal-Ibero, A., Amorn, R., Weilbacher, P.., \& Papaderos, P. 2007, ApJ, 669, 251

Cairós, L. M., Caon, N., Papaderos, P., et al. 2009a, ApJ, 707, 1676

Cairós, L. M., Caon, N., Zurita, C., et al. 2009b, A\&A, 507, 1291

Caon, N., Cairós, L.M., Aguerri, J.A.L., \& Muñoz-Tuñón, C. 2005, ApJS, 157, 218 
Carignan, C., Beaulieu, S., \& Freeman, K.C. 1990, AJ, 99, 178

Ceverino, D., Dekel, A., \& Bournaud, F. 2010, MNRAS, 404, 2151

Chamaraux, P. 1977, A\&A, 60, 67

Contreras Ramos, R., Annibali, F., Fiorentino, G., Tosi, M., Aloisi, A., Clementini, G., Marconi, M., Musella, I., Saha, A., \& van der Marel, R. P. 2011 arXiv1106.5613

Crone, M.M., Schulte-Ladbeck, R.E., Greggio, L., \& Hopp, U. 2002, ApJ, 567, 258

Dalcanton, J.J. \& Stilp, A.M. 2010, ApJ, 721, 547

Del Popolo, A. 2011, MNRAS, tmp.1818

Dohm-Palmer, R.C., Skillman, E.D., Gallagher, J., Tolstoy, E., Mateo, M., Dufour, R.J., Saha, A., Hoessel, J., \& Chiosi, C. 1998, AJ, 116, 1227

Doublier, V., Caulet, A., \& Comte, G. 1999, A\&AS, 138, 213

Drinkwater, M. J., Gregg, M. D., Holman, B. A., \& Brown, M. J. I. 2001, MNRAS, 326, 1076

Ekta, B., Pustilnik, S.A., \& Chengalur, J.N. 2009, MNRAS, 397, 963

Elmegreen, B.G., Bournaud, F., \& Elmegreen, D.M. 2008, ApJ, 688, 67

Elmegreen, B.G., Elmegreen, D.M., Fernandez, M.X., \& Lemonias, J.J., 2009a, ApJ, 692, 12

Elmegreen, D.M., Elmegreen, B.G., Marcus, M., Shahinyan, K., Yau, M., \& Petersen, M. 2009b, ApJ, 701, 306

Elmegreen, B.G. 2011, ApJ, 373, 10

Erb, D. K., Steidel, C. C., Shapley, A. E., Pettini, M., Reddy, N. A., \& Adelberger, K. L. 2006, ApJ, 646, 107

Fellhauer, M., Kroupa, P., and Evans, N.W. 2006, MNRAS, 372, 338

Firpo, V., Bosch, G., Hägele, G.F., Díaz, A.I., \& Morrell, N. 2011, MNRAS. 414, 3288

Förster Schreiber, N. M., Shapley, A. E., Genzel, R., Bouché, N., Cresci, G., Davies, R., Erb, D. K., Genel, S., Lutz, D., Newman, S., Shapiro, K. L., Steidel, C. C., Sternberg, A., \& Tacconi, L. J. 2011, arXiv:1104.0248 
Fricke, K.J., Izotov, Y.I., Papaderos, P., Guseva, N.G., \& Thuan, T.X. 2001, AJ, 121, 169

García-Lorenzo, B., Cairós, L. M., Caon, N., Monreal-Ibero, A., \& Kehrig, C. 2008, ApJ, 677,201

Genel, S., Naab, T., Genzel, R., Förster Schreiber, N.M., Sternberg, A., Oser, L., Johansson, P.H., Davé, R., Oppenheimer, B.D., \& Burkert, A. 2010, arXiv1011.0433

Gil de Paz, A., Zamorano, J., \& Gallego, J. 1999, MNRAS, 306, 975

Goerdt, T., Moore, B., Read, J.I., \& Stadel, J. 2010, ApJ, 725, 1707

Gordon, D., \& Gottesman, S. T. 1981, AJ, 86, 161

Governato, F., Brook, C., Mayer, L., Brooks, A., Rhee, G., Wadsley, J., Jonsson, P., Willman, B., Stinson, G., Quinn, T., \& Madau, P. 2010, Nature, 463, 203

Griffith, R.L., Tsai, C.-W., Stern, D., Blain, A., Eisenhardt, P.R.M., Harrison, F., Jarrett, T.H., Madsen, K., Stanford, S.A., Wright, E.L., Wu, J., Wu, Y., Yan, L. 2011, ApJ,736, L22

Grogin, N.A., \& Geller, M.J. 2000, AJ, 119, 32

Guseva, N. G., Papaderos, P., Izotov, Y. I., Green, R. F., Fricke, K. J., Thuan, T. X., \& Noeske, K. G. 2003, A\&A, 407, 105

Guseva, N. G., Papaderos, P., Izotov, Y. I., Green, R. F., Fricke, K. J., Thuan, T. X., \& Noeske, K. G. 2003, A\&A, 407, 75

Hartmann, M., Debattista, V.P., Seth, A., Cappellari, M., \& Quinn, T.R. 2011, MNRAS, 418, 2697

Heithausen, A., Bensch, F., Stützki, J., Falgarone, E., \& Panis, J. F. 1998, A\&A, 331, L65

Heller, A.B., Brosch, N., Almoznino, E., van Zee, L., Salzer, J.J. 2000, MNRAS, 316, 569

Hoffman, G.L., Brosch, N., Salpeter, E.E., \& Carle, N.J. 2003, AJ, 126, 2774

Hunt, L. K., Dyer, K. K., \& Thuan, T. X. 2005, A\&A, 436, 837

Hunter D.A., Elmegreen, B.G., \& van Woerden, H. 2001, ApJ, 556, 773

Hunter, D.A., \& Elmegreen, B.G. 2004, ApJS, 128, 2170

Hunter, D.A., \& Elmegreen, B.G. 2006, ApJS, 162, 49 
Hunter, D.A. et al. 2011, AJ, in press

Immeli, A., Samland, M., Gerhard, O., \& Westera, P. 2004, A\&A, 413, 547

Inoue, S. 2011, MNRAS, 416, 1181

Izotov, Y. I., Thuan, T. X., \& Lipovetsky, V. 1997, ApJS, 108, 1

Izotov, Y. I., Lipovetsky, V. A., Chaffee, F. H., Foltz, C. B., Guseva, N. G., \& Kniazev, A. Y. 1997, ApJ, 476, 698

Izotov, Y. I., Chaffee, F. H., Foltz, C. B., et al. 2001, ApJ, 566, 222

Izotov, Y. I., Guseva, N. G., Fricke, K. J., \& Papaderos, P. 2009, A\&A, 503, 61

Izotov, Y.I., Thuan, T.X., \& Wilson, J.C. 2009. ApJ, 703, 1984

Izotov, Y.I., Guseva, N.G., Thuan, T.X. 2011, ApJ, 728, 161

Johnson, K.E., Leitherer, C., Vacca, W.D., \& Conti, P.S. 2000, AJ, 120, 1273

Johnson, K.E., Hunt, L.K., \& Reines, A.E. 2009, AJ, 137, 3788

Julian, W.H., \& Toomre, A. 1966, ApJ, 146, 810

Kennicutt, R., Balick, B., \& Heckman, T. 1980, PASP, 92, 134

Koleva, M., Prugniel, P., de Rijcke, S., \& Zeilinger, W.W. 2011, MNRAS, 417, 1643

Kunth, D., Maurogordato, S., \& Vigroux, L. 1988, A\&A, 204, 10

Kuzio de Naray, R. McGaugh, S.S., \& Mihos, J.C. 2009, ApJ, 692, 1321

Lelli, F., Verheijen, M., Fraternali, F., \& Sancisi, R. 2011, arXiv1110.6042

Lo, K.Y., Sargent, W.L.W., \& Young, K. 1993, AJ, 106, 507

Loose, H.-H., \& Thuan, T.X. 1986, ApJ, 309, 59

Loose, H. H., \& Thuan, T. X. 1986, in Star-Forming Dwarf Galaxies and Related Objects, ed. D. Kunth, T. X. Thuan, \& J. Tran Than Van (Gifsur-Yvette: Editions Frontie'res), 73

Lozinskaya, T.A., Moiseev, A.V., Avdeev, V. Yu., \& Egorov, O.V. 2006, Astron. Lett. 32, 361 
Lynden-Bell, D., \& Kalnajs, A. J. 1972, MNRAS, 157, 1

Lynds, R., Tolstoy, E., ONeil, E. J., Jr., \& Hunter, D. A. 1998, AJ, 116, 146

Martin, C. 1997, ApJ, 491, 561

McQuinn, K.B.W., Skillman, E.D., Cannon, J.M., Dalcanton, J., Dolphin, A., HidalgoRodrguez, S., Holtzman, J., Stark, D., Weisz, D., Williams, B. 201, ApJ, 724, 49

Meurer, G.R., Carignan, C., Beaulieu, S.F., \& Freeman, K.C. 1996, AJ, 111, 1551

Navarro, J. F., Frenk, C. S., \& White, S. D. M. 1996, ApJ, 462, 563

Noeske, K.G., Guseva, N.G., Fricke, K.J., Izotov, Y.I., Papaderos, P., \& Thuan, T. X. 2000, A\&A, 361, 33

Noeske, K. G., Papaderos, P., Cairós, L. M., \& Fricke, K. J. 2003, A\&A, 410, 481

Noguchi, M. 1999, ApJ, 514, 77

Ogiya, G. \& Mori, M. 2011, ApJL, 736, 2

Oh, S-H., Brook, C., Governato, F., Brinks, E., Mayer, L., de Blok, W. J. G., Brooks, A., Walter, F. 2011, AJ, 142, 24

Papaderos, P., Loose, H.-H., Fricke, K. J., \& Thuan, T. X. 1996, A\&A, 314, 59

Papaderos, P., Izotov, Y. I., Fricke, K. J., Thuan, T. X., \& Guseva, N. G. 1998, A\&A, 338, 43

Papaderos, P., Fricke, K. J., Thuan, T. X., Izotov, Y. I., \& Nicklas, H. 1999, A\&A, 352, L57

Papaderos, P., Izotov, Y. I., Thuan, T. X., Noeske, K. G., Fricke, K. J., Guseva, N. G., \& Green, R. F. 2002, A\&A, 393, 461

Papaderos, P., Guseva, N. G., Izotov, Y. I., \& Fricke, K. J. 2008, A\&A, 491, 113

Papovich, C., Dickinson, M., Giavalisco, M., Conselice, C.J., \& Ferguson, Henry C. 2005, ApJ, 631, 101

Peimbert, A., \& Peimbert, M. 2010, ApJ, 724, 791

Pringle, J.E. 1981, ARAA, 19, 137

Pustilnik, S. A., Lipovetsky, V. A., Izotov, Yu. I., Brinks, E., Thuan, T. X., Knyazev, A. Yu., Neizvestnyi, S. I., \& Ugryumov, A. V. 1997, AstL, 23, 308 
Pustilnik, S. A., Brinks, E., Thuan, T. X., Lipovetsky, V. A., \& Izotov, Y. I. 2001, AJ, 121, 1413

Pustilnik, S. A., Martin, J.-M., Huchtmeier, W. K., Brosch, N., Lipovetsky, V. A., \& Richter, G. M. 2002, A\&A, 389, 405

Putman, M. E., Bureau, M., Mould, J. R., Staveley-Smith, L., \& Freeman, K. C. 1998, AJ, 115,2345

Ramya, S., Kantharia, N. G., \& Prabhu, T. P. 2011, ApJ, 728, 124

Read, J., \& Gilmore, G. 2005, MNRAS, 356, 107

Read, J. I., Goerdt, T., Moore, B., Pontzen, A. P., Stadel, J., \& Lake, G. 2006, MNRAS, 373,1451

Recchi, S., Matteucci, F., D’Ercole, A., \& Tosi, M. 2004, A\&A, 426, 37

Recchi, S. \& Hensler, G. 2006, A\&A, 445, L39

Revaz, Y., Jablonka, P., Sawala, T., Hill, V., Letarte, B., Irwin, M., Battaglia, G., Helmi, A., Shetrone, M. D., Tolstoy, E., \& Venn, K.A. 2009, A\&A, 501, 18

Roberts, M.S., \& Haynes, M. P. 1994, ARA\&A, 32, 115

Sánchez-Salcedo, F. J., Reyes-Iturbide, J., \& Hernandez, X. 2006, MNRAS, 370, 1829

Sandage, A. 1986, A\&A, 161, 89

Sargent, W. L. W., \& Searle, L. 1970, ApJ, 162, L155

Schroyen, J., de Rijcke, S., Valcke, S., Cloet-Osselaer, A., Dejonghe, H. 2011, MNRAS, 416, 601

Schulte-Ladbeck, R. E., Crone, M.M., \& Hopp, U. 1998, ApJ, 493, L23

Searle, L., \& Sargent, W. L. W. 1972, ApJ, 173, 25

Silich, S., Tenorio-Tagle, G., Muñoz-Tuñón, C., \& Cairos, L. M. 2002, AJ, 123, 2438

Simpson, C.E., et al. 2011, arXiv1107.2049

Spekkens, K., Giovanelli, R., \& Haynes, M.P. 2005, AJ, 129, 2119

Stil, J.M. \& Israel, F.P. 2002, A\&A, 389, 42 
Stinson, G.S., Dalcanton, J.J., Quinn, T., Kaufmann, T., \& Wadsley, J. 2007, ApJ, 667, 170

Stoughton, C., et al. 2002, AJ, 123, 485

Swaters, R. A., van Albada, T. S., van der Hulst, J.M., \& Sancisi, R. 2002, A\&A, 390, 829

Taylor, C., Brinks, E., \& Skillman, E.D. 1993, AJ, 105, 128

Taylor, C.L., Brinks, E., Pogge, R.W., \& Skillman, E.D. 1994, AJ, 107, 971

Taylor, C.L., Thomas, D.L., Brinks, E., \& Skillman, E.D. 1996, ApJS, 107, 143

Terlevich, R., \& Melnick, J. 1981, MNRAS, 195, 839

Thuan, T.X. 1983, ApJ, 268, 667

Thuan, T. X., \& Martin, G. E. 1981, ApJ, 247, 823

Thuan, T.X., \& Izotov, Y.I. 1997, ApJ, 489, 623

Thuan, T. X., Izotov, Y. I., \& Lipovetsky, V. A. 1997, ApJ, 477, 661

Thuan, T.X., Izotov, Y.I., \& Foltz, C.B. 1999, ApJ, 525, 105

Thuan, T.X., Hibbard, J.E., \& Levrier, F. 2004, AJ, 128, 617

Valenzuela, O., Rhee, G., Klypin, A., Governato, F., Stinson, G., Quinn, T., \& Wadsley, J. 2007, ApJ, 657, 773

van Eymeren, J., Trachternach, C., Koribalski, B. S., \& Dettmar, R.-J. 2009, A\&A, 505, 1

van Zee, L., Westpfahl, D., Haynes M.P. \& Salzer, J.J. 1998, AJ, 115, 1000

van Zee, L., Skillman, E.D., \& Salzer, J.J. 1998, 1998, AJ, 116, 1186

van Zee, L., Salzer, J.J., \& Skillman, E.D. 2001, AJ, 122, 121

Wang, J.-M., Yan, C.-S., Li, Y.-R., Chen, Y.-M., Xiang, F., Hu, C., Ge, J.-Q., \& Zhang, S. 2009, ApJL, 701, 7

Wilcots, E.M. \& Miller, B.W. 1998, AJ, 116, 2363

Zackrisson, E., Bergvall, N., Marquart, T., \& Östlin, G. 2006, A\&A, 452, 857

Zhang, H.-X., Hunter, D.A., Elmegreen, B.G., Gao, Y., \& Schruba, A. 2011, arXiv:1111.3363 
Zhang, X., \& Buta, R.J. 2007, in Galaxy Evolution Across the Hubble Time, eds F. Combes \& J. Palous, UAU Symposium 235, 184

Zhao, Y., Gu, Q., \& Gao, Y. 2011, AJ, 141, 68 
Table 1. Sample BCD Galaxies and their Clump Properties ${ }^{\mathrm{a}}$

\begin{tabular}{lcccccccccc}
\hline \hline Galaxy & $\begin{array}{c}\mathrm{D} \\
\mathrm{Mpc}\end{array}$ & $\begin{array}{c}\log M_{\mathrm{s}} \\
M_{\odot}\end{array}$ & $\begin{array}{c}\log M_{\mathrm{b}} \\
M_{\odot}\end{array}$ & $\begin{array}{c}\log M_{\mathrm{c}} \\
M_{\odot}\end{array}$ & $\begin{array}{c}r_{\mathrm{c}} \\
\mathrm{kpc}\end{array}$ & $\begin{array}{c}\text { Aperture } \\
\mathrm{kpc}\end{array}$ & $\begin{array}{c}\log M_{\mathrm{dyn}}\left(r_{\mathrm{c}}\right) \\
M_{\odot}\end{array}$ & $\begin{array}{c}M_{\mathrm{c}} / M_{\mathrm{dyn}}\left(r_{\mathrm{c}}\right) \\
\ln \Lambda\end{array}$ & $\begin{array}{c}T \\
\mathrm{Gyr}\end{array}$ \\
\hline Mrk 178 & 3.9 & 7.04 & 7.39 & 5.13 & 0.39 & 0.32 & 6.60 & 0.035 & $3.4-4.3$ & $0.50-1.4$ \\
DDO 155 & 2.2 & 6.47 & 7.22 & 5.46 & 0.21 & 0.24 & 6.78 & 0.048 & $3.2-3.9$ & $0.12-0.37$ \\
Haro 29 & 5.9 & 7.16 & 8.06 & 6.33 & 0.27 & 0.74 & 6.26 & 1.17 & $1.3-2.3$ & $0.03-0.06$ \\
NGC 2366 & 3.4 & 7.84 & 9.04 & 6.23 & 1.31 & 0.93 & 8.08 & 0.014 & $3.6-4.0$ & $1.3-4.2$ \\
NGC 4861 & 7.6 & 8.04 & 8.83 & 6.89 & 2.07 & 0.67 & 8.48 & 0.026 & $1.8-1.8$ & $1.7-6.3$ \\
\hline
\end{tabular}

${ }^{a} D$ is the distance, $M_{\mathrm{s}}$ is the galaxy stellar mass, $M_{\mathrm{b}}$ is the galaxy baryonic mass, $M_{\mathrm{c}}$ is the clump stellar mass, $r_{c}$ is the clump galacticentric radius, Aperture is the aperture size used for clump photometry, $M_{\mathrm{dyn}}$ is the galaxy dynamical mass inside $r_{\mathrm{c}}, \Lambda$ is the Coulomb factor, and $T$ is the clump accretion time. For the latter two, we assume $\xi=0.2$ and a rotation curve slope $\beta=0.5$ in the first case (NFW core), and $\xi=0.03, \beta=1$ in the second case (Burkert core), with factors of 0.40 and 0.33 in $T$, respectively, to account for the decrease in $M_{\mathrm{dyn}}$ with radius. 
Table 2. Inner Scale Heights and Lengths ${ }^{a}$

\begin{tabular}{lcccc}
\hline \hline Galaxy & $\begin{array}{c}\Sigma \\
M_{\odot} \mathrm{pc}^{-2}\end{array}$ & $\begin{array}{c}H \\
\mathrm{kpc}\end{array}$ & $\begin{array}{c}R_{\mathrm{d}} \\
\mathrm{kpc}\end{array}$ & $H / R_{\mathrm{d}}$ \\
\hline Mrk 178 & 4.9 & 1.8 & 0.27 & 6.7 \\
DDO 155 & 7.4 & 1.0 & 0.22 & 4.5 \\
Haro 29 & 39 & 0.19 & 0.20 & 0.95 \\
NGC 2366 & 0.66 & 1.3 & 3.7 & 0.35 \\
NGC 4861 & 10 & 0.74 & 1.0 & 0.74 \\
\hline
\end{tabular}

${ }^{a} H$ is the inner disk scale height assuming a perpendicular velocity dispersion of $10 \mathrm{~km} \mathrm{~s}^{-1}$ and the observed stellar mass column density, $\Sigma ; R_{\mathrm{d}}$ is the inner disk scalelength. 


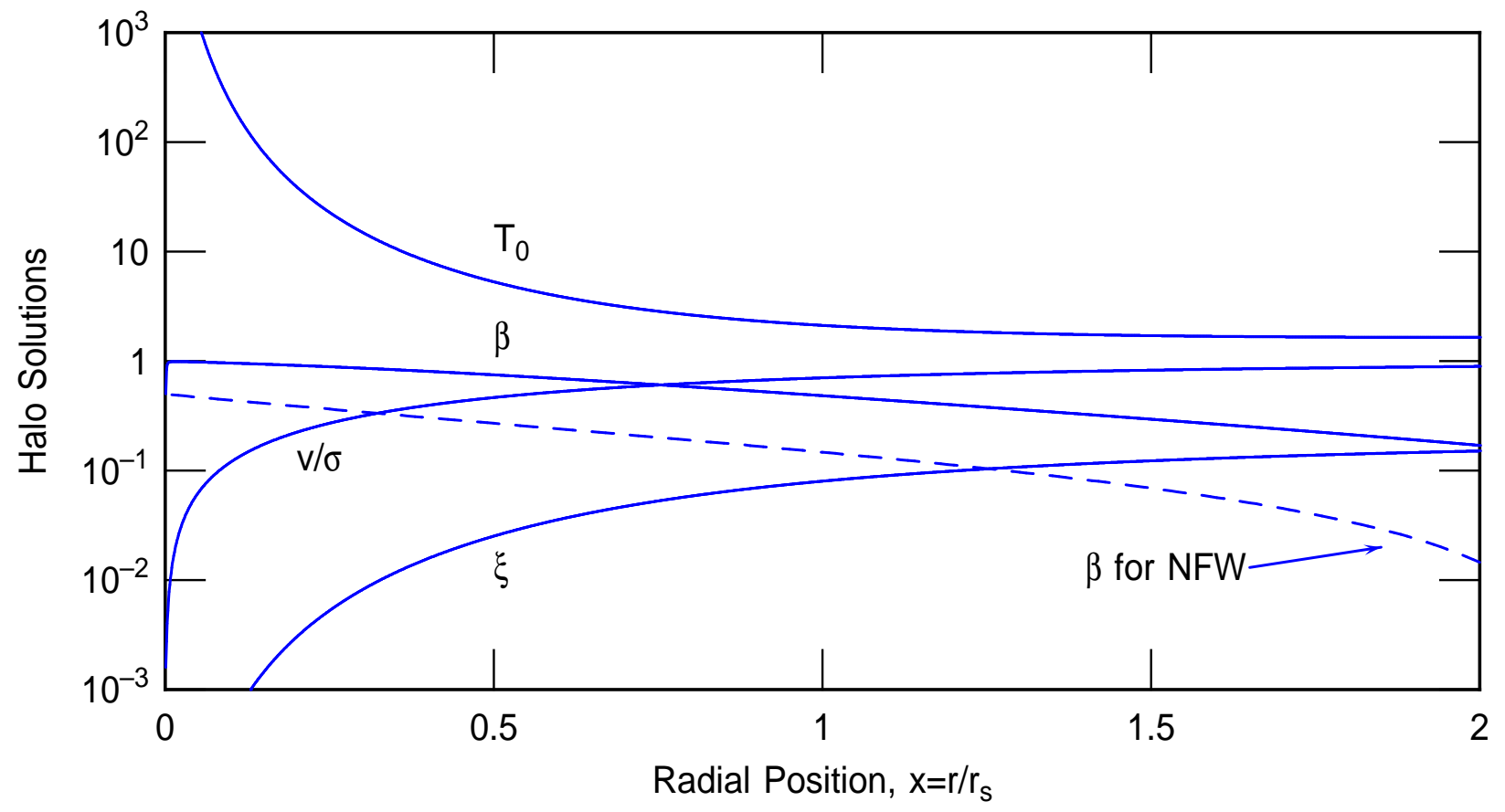

Fig. 1. - Solutions to various parameters connected with the Burkert (1995) dark matter density profile, which has a constant-density core; $\xi$ is the dynamical friction parameter in the parentheses of equation 1, $v$ is the rotation speed, $\sigma$ is the $3 \mathrm{D}$ velocity dispersion, $\beta$ is the slope of the rotation curve, and $T_{0}$ is the prefactor in equation 2 . The dashed line shows $\beta$ for a Navarro et al. (1996) profile. 


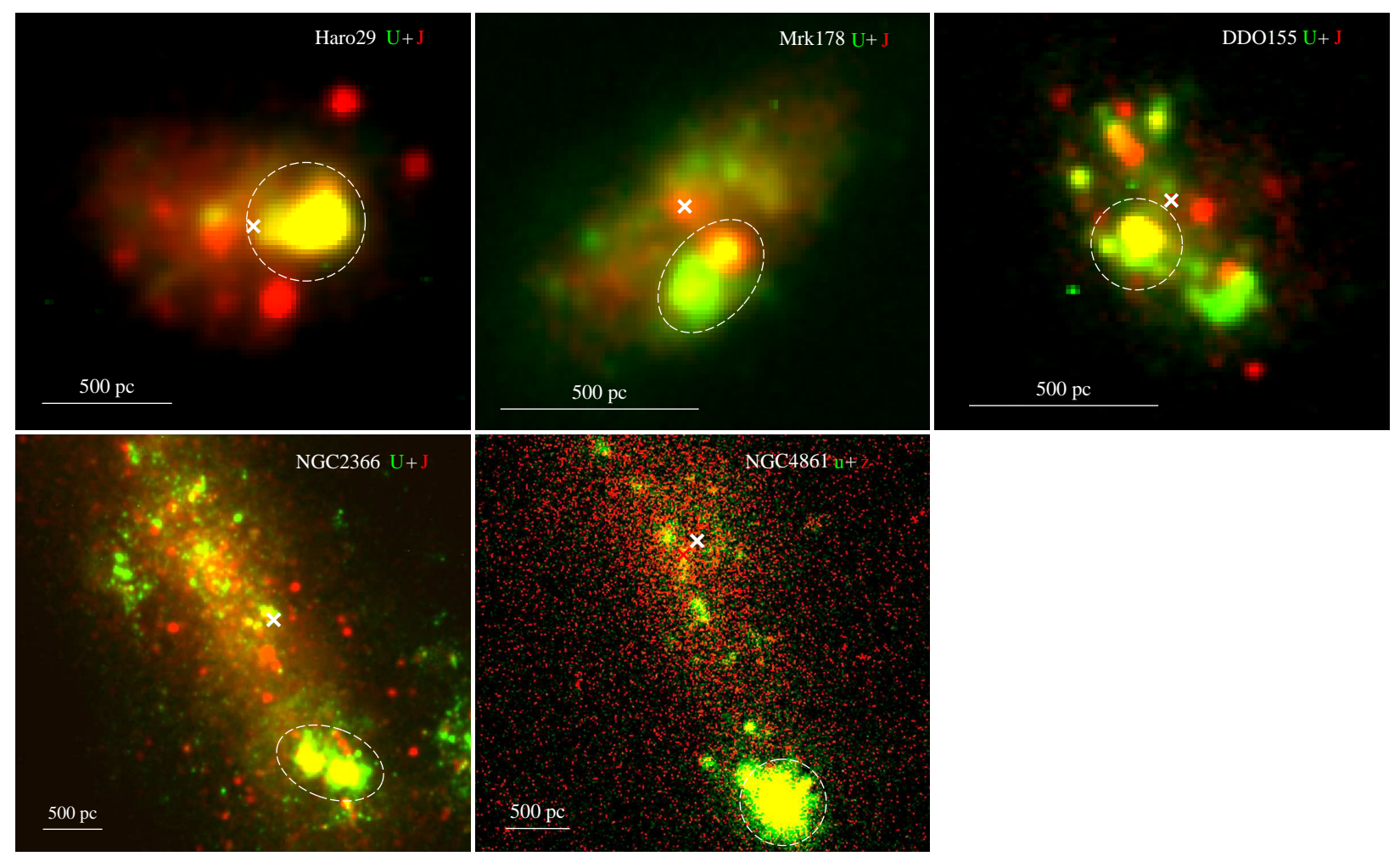

Fig. 2.- Five clumpy irregular galaxies used to study relative clump mass and possible central migration from tidal torques. The images are a combination of $\mathrm{U}$ and J-band from Hunter \& Elmegreen (2006) except for NGC 4861, which is a combination of $\mathrm{u}$ and z-band from SDSS. The measured clumps are indicated by elliptical contours and the centers of the outer V-band isophotes are indicated by " $\mathrm{x}$ ". 